\title{
Implementation of a Novel Concatenated FEC by RS and Irregular Turbo Codes on OFDM Systems
}

\author{
${ }^{1}$ A. Shanmugam, ${ }^{2}$ A.R. Abdul Rajak, ${ }^{2}$ S. Vishwanath and ${ }^{2}$ R. Upendra \\ ${ }^{1}$ Bannariamman Institute of Technology, Erode, Tamil Nadu, India \\ ${ }^{2}$ PSG College of Technology, Coimbatore, Tamil Nadu, India
}

\begin{abstract}
Code Division Multiple Access (CDMA) performs well in a multi-cellular environment where a single frequency is used in all cells. Code Division Multiple Access (CDMA) has severe problems associated with multipath and Channel news. One possible problem is that the receiver may require a very large dynamic range in order to handle the large signal strength variation among users. Due to that the Bit Error Rate (BER) is getting increased. Moreover due to the addition of uncontrollable random channel noise degrade the performance of the receiver. The multipath problems give inter symbol interference. This can be reduced by Orthogonal Frequency Division Multiplexing (OFDM) technique. More work could be done on investigating suitable techniques for doing OFDM promises to be a suitable modulation technique for high capacity wireless communications and will become increasing important in the future as wireless networks become more relied on. In order to increase the wireless network performance, this study has concentrated on OFDM with a novel forward error correction by data transmission with Quadrature Phase Shift Keying (QPSK) as modulation techniques. Here an FEC scheme by concatenation of Reed Solomon (RS) and the Irregular Turbo code is implemented in OFDM. The simulation results show that the system BER performance is decreased with this technique, when compared to the system having Forward Error Correction (FEC) by RS code alone and irregular turbo code alone.
\end{abstract}

Key words: CDMA, OFDM, BER, FEC, QPSK, ISDN, GSM, UMTS, FDMA, TDMA

\section{INTRODUCTION}

The expansion of the use of digital networks has led to the need for the design of new higher capacity communications networks. The demand for cellulartype systems is getting increased day by day. Wireless services have been growing at a rate greater than $50 \%$ per year, with the current second-generation Global Service for Mobile Communication (GSM) being expected to be filled to capacity. Integrated Service and Digital Network (ISDN) the increased reliance on computer networking and the Internet has resulted in demand for connectivity to be provided "anywhere, any time", leading to an increase in the demand for wireless systems. This demand has driven the need to develop new high capacity, high reliability wireless telecommunications systems. The telecommunications industry is also changing, with a demand for a greater range of services such as video conferencing, Internet services and data networks and multimedia. This demand for higher capacity networks has led to the development of third generation telecommunications systems. One of the proposed third generation telecommunications systems is the Universal Mobile Telecommunications System (UMTS), which aims to provide a more flexible data rate, a higher capacity and a more tightly integrated service, than current second generation mobile systems. The development and deployment of third generation telecommunication systems aim to overcome some of the downfalls of current wireless systems by providing a high capacity, integrated wireless. These wireless systems should have maximum spectrum efficiency and should have good and satisfactory performance. Hence $4 \mathrm{G}$ networks have OFDM as modulation technique

\section{THE OFDM SYSTEMS}

The Orthogonal Frequency Division Multiplexing (OFDM) technique ${ }^{[1-3]}$ divides the frequency spectrum available into many closely spaced carriers, which are individually modulated by low-rate data streams. In this sense, OFDM is similar to Frequency Division Multiple Access (FDMA) (The bandwidth is divided into many channels, so that, in a multi-user environment, each channel is allocated to a user). However, the difference lies in the fact that the carriers chosen in OFDM are much closer spaced than in FDMA $(1 \mathrm{kHz}$ in OFDM as opposed to about $30 \mathrm{kHz}$ in FDMA), thereby increasing its spectral usage efficiency. The orthogonality between the carriers is what facilitates the close spacing of carriers. The orthogonality principle essentially implies that each carrier has a null at the center frequency of each of the other carriers in the system while also maintaining an integer number of cycles over a symbol period. The motivation for using OFDM techniques over Time Division Multiple Access (TDMA) techniques is twofold. First, TDMA limits the total 
number of users that can be sent efficiently over a channel. In addition, since the symbol rate of each channel is high, problems with multipath delay spread invariably occur. In stark contrast, each carrier in an OFDM signal has a very narrow bandwidth (i.e. $1 \mathrm{kHz}$ ); thus the resulting symbol rate is low. This result in the signal having a high degree of tolerance to multipath delay spread, as the delay spread must be very $\log$ to cause significant inter-symbol interference (e.g.>500usec).

\section{OFDM SYSTEM WITH A NOVEL FEC SCHEME BY CONCATENATION OF RS CODE AND IRREGULAR TURBO CODE}

The OFDM system performance can be increased by employing FEC. RS and Irregular Turbo Codes are considered. The reason for choosing RS code is that it can correct a number of error bits due to burst noise. Since irregular Turbo code ${ }^{[4-9]}$ performs better among all the code, so it is recommended for its use in OFDM system. To improve the system performance still, the only solution required is that implement the concatenated code ${ }^{[10-13]}$. Here two or more codes are concatenated to improve the performance. In this study, a Novel FEC scheme by Concatenation of RS code and Irregular Turbo code is implemented in OFDM system as shown in Fig. 1. Here the RS code is made as inner code and irregular turbo code as outer code.

\section{Reed-Solomon Code:}

RS Encoder: The generator polynomial for an RS code takes following form as given eq. (1):

$$
g(X)=g_{0}+g_{1} X+g_{2} X^{2}+\ldots . .+g_{2 t-1} X^{2 t-1}+X^{2 t}
$$

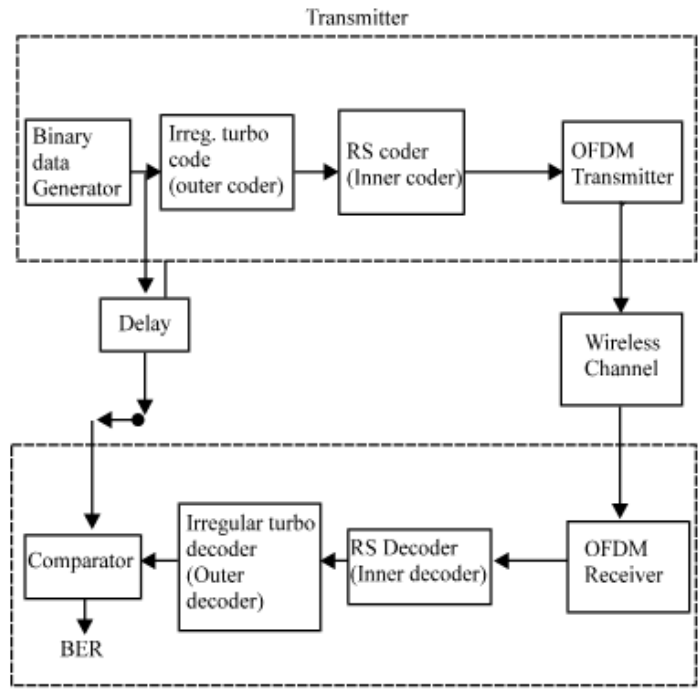

Receiver

Fig. 1: A Novel FEC Scheme for OFDM System
The degree of the polynomial is equal to number of parity symbols. If $\alpha$ is the primitive element in GF $\left(2^{\mathrm{m}}\right)$ then generator polynomial ${ }^{[15-17]}$ can be described as Eq. (2):

$g(X)=(X-\alpha)\left(X-\alpha^{2}\right) \ldots .\left(X-\alpha^{2 t}\right)$

Let $\mathrm{m}(\mathrm{X})=\mathrm{m}_{0}+\mathrm{m}_{1}(\mathrm{X})+\ldots \ldots \mathrm{m}_{\mathrm{k}-1}(\mathrm{X})$ is the message polynomial. The encoding is performed by shifting the message polynomial $m(x)$ into the rightmost $\mathrm{k}$ stages of the codeword register and then appending a parity polynomial $\mathrm{p}(\mathrm{x})$ by placing it in the leftmost $\mathrm{n}-\mathrm{k}$ stages. Mathematically the encoding process is described as eq. (3):

$X^{n-k} m(X)=q(X) g(X)+p(X)$

where, $\mathrm{p}(\mathrm{X})$ and $\mathrm{q}(\mathrm{X})$ are remainder and quotient polynomial respectively, eq. (3) can be written as eq. (4):

$\mathrm{p}(\mathrm{X})=\mathrm{X}^{\mathrm{n}-\mathrm{k}} \mathrm{m}(\mathrm{X})$ modulo $\mathrm{g}(\mathrm{X})$

The resulting code word is formed by concatenating $\mathrm{p}(\mathrm{X})$ with $\mathrm{m}(\mathrm{X})$. The encoding can be performed by (n-k) shift register. After an entire block of input is given to the above circuit, the shift registers contain the parity symbols. These are multiplexed to the input data to form the coded sequence.

RS Decoder: Let $\mathrm{v}(\mathrm{x})=\mathrm{v}_{0}+\mathrm{v}_{1} \mathrm{x}+\ldots \ldots \ldots+\mathrm{v}_{\mathrm{n}-1} \mathrm{x}^{\mathrm{n}-1} \quad$ be the transmitted code vector and $\mathrm{r}(\mathrm{x})=\mathrm{r}_{0}+\mathrm{r}_{1} \mathrm{x}+\ldots \ldots+\mathrm{r}_{\mathrm{n}-1} \mathrm{x}^{\mathrm{n}-1}$ be the corresponding received vector. Then the error pattern added by the channel is $\mathrm{e}(\mathrm{x})=\mathrm{r}(\mathrm{x})-\mathrm{V}(\mathrm{x})=\mathrm{e}_{0}+\mathrm{e}_{1} \mathrm{x}+\ldots \ldots \ldots+\mathrm{e}_{\mathrm{n}-1} \mathrm{x}^{\mathrm{n}-1}$.

The function of the decoder is to find out the error pattern so that actual transmitted vector can be found from the received vector as in eq. (5):

$\mathrm{v}(\mathrm{x})=\mathrm{r}(\mathrm{x})+\mathrm{e}(\mathrm{x})$

Assuming the received vector has $\mathrm{V}$ errors in positions $i_{1}, i_{2}, i_{3} \ldots . i_{v}$. Then e ( $\left.x\right)$ is given as in eq. (6):

$e(x)=e_{i 1} x^{i 1}+e_{i} x^{i 2}+\ldots \ldots+e_{i v} x^{i v}$

Hence, to determine the e (x), error locations and error values should be calculated. So the RS decoding steps consist of following steps:

* Computation of syndrome 


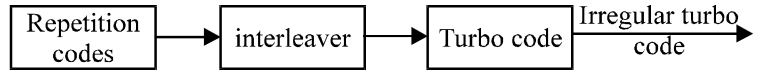

Fig. 2: General form of Irregular Turbo Code

* Finding error locator polynomial

* Finding roots of an error locator polynomial

* Calculation of error magnitudes

\section{Irregular Turbo Code:}

Irregular Turbo Code Structure: The structure of the irregular turbo $\operatorname{code}^{[14-16]}$ is slightly different from normal turbo code. By slight modifications, the original turbo can be made irregular ${ }^{[17]}$. A turbo code can be made as irregular. Figure 2 shows the set of systematic bits (middle rows of discs) being fed directly into one convolutional code (the chain at the bottom) since the order of the systematic bits is irrelevant, an interviewer may be introduced before the upper convolutional code. For long codes, the values of initial state and final state of the conventional code do not significantly influence the performance. So turbo code may be viewed as a code that copies the systematic bits, interleaves both sets of these bits and then feeds them into conventional code. Some systematic bits may be tied together, in effect causing some systematic bits to be replicated more than once. Even though, some bits are repeated, some bits are punctured to make overall rate fixed. A more general form of irregular turbo code can be obtained by a cascade of array of repetition codes, an interleaver and a turbo code.

Decoding of Irregular Turbo Codes: The decoding scheme used for irregular turbo code is slightly different from original turbo decoding because of the employment of an interleaver. The decoding algorithm is as follows:

* After receiving channel output, the channel output $\log$ likelihood ratios for the $\mathrm{N}$ codeword bits $\left(\mathrm{L}_{1}^{0}, \mathrm{~L}_{2}^{0}, \ldots \ldots, \mathrm{L}_{\mathrm{N}}^{0}\right)$ is computed.

* If the codeword bit i have degree $\mathrm{d}$, then $\log$ likelihood ratios are assigned as in eq. (10):
$\mathrm{L}_{\mathrm{i}, 1} \leftarrow \mathrm{L}_{\mathrm{i}}^{0}, \mathrm{~L}_{\mathrm{i}, 2} \leftarrow \mathrm{L}_{\mathrm{i}}^{0} \ldots . . \mathrm{L}_{\mathrm{i}, \mathrm{d}} \leftarrow \mathrm{L}_{\mathrm{i}}^{0}$

The likely hood ratios are interleaved and fed for Logarithmic Maximum a Posterior probability (logMAP) algorithm. The Map algorithm assumes that the inputs are priori log probability ratios and then computes a set of posteriori log-probability ratios $L_{i, 1}^{\prime}, L_{i, 2}^{\prime}, \ldots . L_{i, d}^{\prime}$. For regular code turbo code, there are just two a posteriori probability ratios $\mathrm{L}_{\mathrm{i}, 1}^{\prime}$ and $_{L_{i, d}^{\prime}}$, for each they correspond to the extrinsic information produced by each constituent convolutional code

* The current estimate of the log-probability ratio for a bit $i$ have given the channel output is given as in eq. (11)

$\hat{\mathrm{L}}_{\mathrm{i}} \leftarrow \mathrm{L}_{\mathrm{i}}^{0}+\sum_{\mathrm{k}=1}^{\mathrm{d}}\left(\mathrm{L}_{\mathrm{i}, \mathrm{k}}^{\prime}-\mathrm{L}_{\mathrm{i}, \mathrm{k}}\right)$

* To compute the inputs to the MAP algorithm needed for next iteration, subtraction off corresponding outputs from the MAP algorithm produced by the previous iteration as in eq. (12)

$\mathrm{L}_{\mathrm{i}, \mathrm{k}} \leftarrow \hat{\mathrm{L}}_{\mathrm{i}}-\mathrm{L}_{\mathrm{i}, \mathrm{k}}^{\prime}$

This completes the first iteration. The steps from 1 to 4 are repeated for required iterations and taking repetitions into account to combine the outputs to form estimates of the log-probability ratios of the codeword.

\section{SIMULATION RESULTS}

The performance concatenated RS and irregular turbo codes are analyzed using data frame sizes of 460 , which is close to the GSM standard (456 bits) for both AWGN and RAYLEIGH channels. All the simulations were performed in MATLAB 6.1.

\section{Simulation Parameters:}

Frame size

Generator polynomial

Interleave size

Decoding iterations

Encoded length (n)

Channels: AWGN and Rayleigh,

Irregularity type

Irregularity percentage

Interleave in Irregular turbo code

Decoding algorithm in Irregular turbo code
: 460 bits, Number of frame repetitions

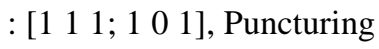

: Golden (size 460 bits), Decoding algorithm

$: 3$, Field used: GF $\left(2^{5}\right)$, Message length (k)

$: 31$, Error correcting capability (t)

: pseudo-random

: $\{5,10$ and $15 \%\}$

Golden

Log-Map.
: 100

: unpunctured

: Log MAP

$: 23$

: 4 symbols 

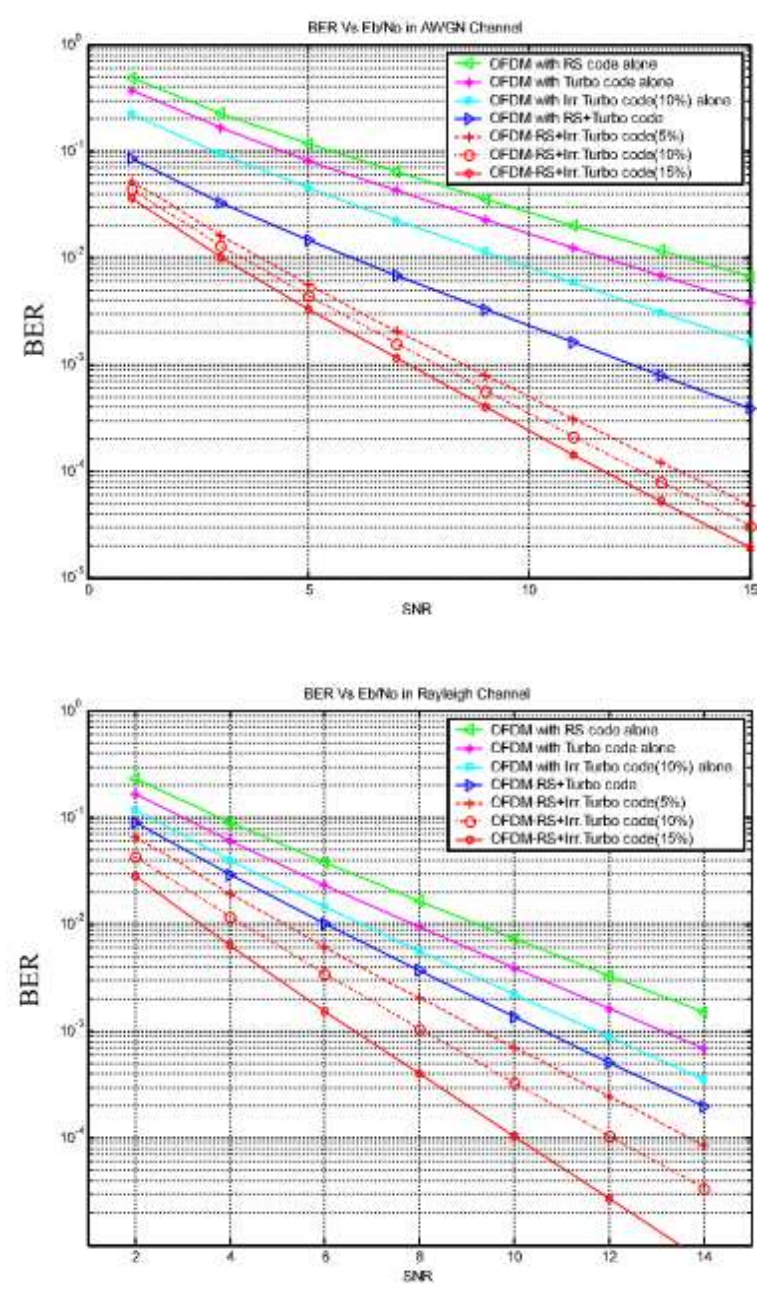

Fig. 5: Performance of Concatenated Code on OFDM Systems

Figure 5 shows the BER performance of OFDM system with the FEC by individual codes and the FEC by concatenated codes on AWGN Channel and Rayleigh Channel. From the performance characteristics, it is clearly shown that that concatenated FEC by RS+Irregular Turbo code exhibits better performance in all other codes.

Results comparison has the lowest BER of just greater than $3 \times 10^{-3}$. The difference in their BERs is greater at an SNR of $10 \mathrm{~dB}$ with RS codes exhibiting a BER just less than $3 \times 10^{-2}$ and the concatenated RS $+15 \%$ irregular turbo code exhibiting $2 \times 10^{-4}$. Similarly in Rayleigh channel at an SNR of $2 \mathrm{~dB}$, RS code exhibits the highest BER just greater than $2 \times 10^{-1}$. Concatenation of RS and 15\% irregular turbo gives the lowest BER of just less than $3 \times 10^{-2}$. At an SNR of 12 $\mathrm{dB}$, RS code gives a BER of $3 \times 10^{-3}$, while concatenated $\mathrm{RS}+15 \%$ irregular turbo codes gives the lowest BER of $3 \times 10^{-5}$.
Table 1: BER Performance of OFDM Systems in AWGN Channel

\begin{tabular}{lll}
\hline & SNR $=5 \mathrm{~dB}$ & $\mathrm{SNR}=10 \mathrm{~dB}$ \\
Code & BER & BER \\
\hline RS & $1 \times 10^{-1}$ & $3 \times 10^{-2}$ \\
Irregular Turbo $(10 \%)$ & $8 \times 10^{-2}$ & $8 \times 10^{-3}$ \\
RS+turbo & $1 \times 10^{-2}$ & $2 \times 10^{-3}$ \\
RS+irr.Turbo(10\%) & $4 \times 10^{-3}$ & $3 \times 10^{-4}$ \\
Rs+Irr.Turbo(15\%) & $3 \times 10^{-3}$ & $2 \times 10^{-4}$ \\
\hline
\end{tabular}

In AWGN channel at an SNR of $5 \mathrm{~dB}, \mathrm{RS}$ codes exhibit the highest BER which is just greater than $1 \times 10^{-1}$. Whereas concatenated RS and 15\% irregular turbo code

Table 2: BER Performance of OFDM Systems in Rayleigh Channel

\begin{tabular}{lcc}
\hline Code & $\begin{array}{l}\text { SNR }=2 \mathrm{~dB} \\
\text { BER }\end{array}$ & $\begin{array}{l}\text { SNR=12 dB } \\
\text { BER }\end{array}$ \\
\hline RS & $2 \times 10^{-1}$ & $3 \times 10^{-3}$ \\
Irregular Turbo (10\%) & $1 \times 10^{-1}$ & $9 \times 10^{-4}$ \\
RS+turbo & $9 \times 10^{-2}$ & $5 \times 10^{-4}$ \\
RS+irr.Turbo(10\%) & $4 \times 10^{-2}$ & $1 \times 10^{-4}$ \\
Rs+Irr.Turbo(15\%) & $3 \times 10^{-2}$ & $3 \times 10^{-5}$ \\
\hline
\end{tabular}

\section{CONCLUSION AND FUTURE DIRECTIONS}

From above plots it is clear that concatenated turbo-RS code has a performance better than that of original turbo code. The irregular turbo code is better than turbo code. Finally concatenated irregular turbo and RS code has a better performance of all codes and thus it can be suggested for wireless communication. Since the OFDM system having we proposed a novel FEC scheme by concatenation of RS and irregular turbo code exhibits best performance in terms of BER, it can be implemented in wireless networks having any application.

Future Directions: This concatenation scheme can also be implemented in CDMA-OFDM systems and the performance characteristics can be studied.

\section{REFERENCES}

1. Richard Van Nee and Ramjee Prasad, 2000. FDM for Wireless Multimedia Communications. Artech House Publisher, Boston.

2. Weinstein, S.B., 1971. Data Transmission by Frequency-Division Multiplexing Using the Discrete Fourier Transform. IEEE Trans. Commune, 19: 628-3.

3. Orthogonal Frequency Division Multiplexing (OFDM) Explained, Magis Networks, Inc., 2001, www.magisnet works.com 
4. Crozier, S., J. Lodge, P. Gunand and A. Hunt, 1999. Performance of Turbo-Codes with Relative Prime and Golden Interleaving Strategies. $6^{\text {th }}$ Intl. Mobile Satellite Conference (IMSC'99), Ottawa, Canada, pp: 268-275.

5. Hagenauer, J. and P. Robertson, 1994. Iterative Turbo Decoding of Systematic convolutional Codes with MAP and SOVA algorithms. In Proc. ITG Conference on Source and Channel Coding, Munich.

6. Lance C. Perez, 1996. A distance spectrum interpretation of turbo code. IEEE Transactions on Information Theory, vol.: 42.

7. Sergio Benedetto, 1996. Unveiling turbo codes: Some results on parallel concatenated code. IEEE Transactions on Information Theory, vol.: 42.

8. Joachim Hagenauer, 1997. The turbo code principle: Tutorial introduction and state of Art. Intl. Symposium on Turbo Codes, France.

9. Ken Gracie, Stewart Crozier, Andrew Hunt and John Lodge. The performance of the low complexity turbo decoder and its implementation on a low-cost, 16-bit fixed point DSP. Communication Research Center, Ottawa.

10. Zhang Zhongpei and Zhou Liang. A low complex parallel decoding scheme for turbo codes. Dept. Of UESTC, Chedgdu, China.
11. Hagenauer, J., E. Offer and L. Papke, 1996. Iterative decoding of binary block and convolutional codes. IEEE Transactions on Information Theory, vol.: 42.

12. Benedetto, S. And G. Montorsi, 1997. Generalized concatenated codes with interleaves. In Proc. Intl. Symposium on Turbo Codes and Related Topics, Brest, France.

13. Le Nhat and R.M.A.P Rajatheva. The performance of parallel concatenated code convolutional codes and serial concatenated codes in AWGN channel. Institute of Technology, Bangkok.

14. Frey, B.J. and D.J.C Mackay, 1999. Irregular turbo codes. Proc. $37^{\text {th }}$ Allerton Conference on Communication, Control and Computing, Allerton House, Illinois.

15. Joseph J. Bosutrous, 2001. Asymptotic behavior study of irregular turbo codes, China.

16. Alex Hubner, 2001. Irregular turbo codes and unequal error protection. Dept. Of Telecommunications, University of ULM, Washington.

17. Simon Haykin, Communication Systems. John Willey and Sons, $4^{\text {th }}$ Edn. 